“C 2017 IEEE. Personal use of this material is permitted. Permission from IEEE must be obtained for all other uses, in any current or future media, including reprinting/republishing this material for advertising or promotional purposes, creating new collective works, for resale or redistribution to servers or lists, or reuse of any copyrighted component of this work in other works." 


\title{
Real-Time WebRTC-Based Design for a Telepresence Wheelchair
}

\author{
Van Kha Ly Ha, Student Member, EMBS, IEEE, Rifai Chai, Member, IEEE, \\ and Hung T. Nguyen, Senior Member, IEEE
}

\begin{abstract}
This paper presents a novel approach to the telepresence wheelchair system which is capable of real-time video communication and remote interaction. The investigation of this emerging technology aims at providing a low-cost and efficient way for assisted-living of people with disabilities. The proposed system has been designed and developed by deploying the JavaScript with Hyper Text Markup Language 5 (HTML5) and Web Real-time Communication (WebRTC) in which the adaptive rate control algorithm for video transmission is invoked. We conducted experiments in real-world environments, and the wheelchair was controlled from a distance using the Internet browser to compare with existing methods. The results show that the adaptively encoded video streaming rate matches the available bandwidth. The video streaming is high-quality with approximately 30 frames per second (fps) and round trip time less than 20 milliseconds (ms). These performance results confirm that the WebRTC approach is a potential method for developing a telepresence wheelchair system.
\end{abstract}

\section{INTRODUCTION}

The advancement of telepresence technologies has revolutionized the way people communicate in daily life. Telepresence systems refer to a set of technologies which are capable of providing video conferencing and remote interaction to give participants a sense of being present at a distant place. Researchers have recently developed various prototypes for telepresence systems. Typical examples of such works included [1-3] in which the authors developed telepresence robots based on existing video conferencing platforms using Voice over Internet Protocol (VoIP) and Skype platform [1-3].

In the development of the emerging information technology, a so-called Web Real-time Communication (WebRTC). WebRTC has offered a powerful technology that facilitates two-way video communication between browsers and provided an efficient way for real-time communications [4]. WebRTC is a standard that defines a set of communication protocols and provides a free open source for developing real-time applications without the need of plugins or software installation. Some research about the protocols and methods for developing real-time applications has been started since the first version of WebRTC was released by Google [4]. Besides, there has been a significant increase in the investigation into WebRTC for healthcare delivery.

Van Kha Ly Ha, Rifai Chai, Hung T. Nguyen are with the Centre for Health Technologies, Faculty of Engineering and Information Technology, University of Technology Sydney, NSW 2007, Australia.

(E-mail: VanKha.L.Ha@student.uts.edu.au; Rifai.chai@uts.edu.au; Hung.Nguyen@uts.edu.au).
Much research has been developed the use of WebRTC in healthcare applications to provide a better quality of life for the elderly and individuals with disabilities. For instance, Chiang, et al. [5] presented a video conferencing system based on WebRTC for seniors while Web shopping for older adults was also presented in [6]. However, to the best of our knowledge, there has been no previous work which uses WebRTC for a telepresence wheelchair to assist wheelchair users for healthcare purposes. The use of telepresence wheelchairs is to provide the flexibility of controlling the wheelchairs by users when their carers are not available.

Our research group has recently demonstrated a telepresence wheelchair based on the Internet protocols and the Skype cross-platforms [3, 7]. Our prior attempts have achieved some degrees of success in software and hardware development. However, its limitation is the processing and handling time of third-party plugins. Moreover, the protocols of the existing platform are unpublicized. Therefore, it is highly desirable to develop an independent application to extend the advanced functionality of the system.

Motivated by the above observation, this paper proposes new development in emerging information technology for an assistive system for healthcare applications. Compared to the previous works $[3,7]$, the current work invokes the novel video communication framework to improve the performance of a telepresence wheelchair system further. More specifically, the aim of this study is to present a novel technical solution to the telepresence wheelchair system based on WebRTC for people with disabilities.

In this study, we have explored and made a first attempt to develop an Internet-enabled wheelchair using WebRTC technology for two-way video interaction and remote controllability in real-time. In the framework development and implementation phase, we adopted the Google Congestion Control (GCC) algorithm to adapt the video transmission rate to the available bandwidth and improve the quality of data transmission. In order to demonstrate the effectiveness of the proposed approach for such a telepresence wheelchair system, real-world experiments were conducted to enable the remote user to drive the wheelchair via the Internet browsers.

The rest of this paper is organized as follows. Section II describes the methodology including real-time web communication and the design of telepresence wheelchair. Section III is devoted to the experiments, results, and discussions. Finally, conclusions and future works are drawn in Section IV. 


\section{METHODOLOGY}

\section{A. Web Real-Time Communication}

The WebRTC has been developed and released by Google as an open source. It was standardized by the Internet Engineering Task Force (IETF) and the World Wide Web Consortium (W3C) [8, 9]. IETF is standardizing protocols used in WebRTC, while JavaScript application programming interfaces (APIs) implemented in Web browsers is being standardized by W3C [9]. WebRTC aims to enable real-time communications between Web browsers, without requiring any plugins. In our development, we have employed the following key components of WebRTC:

MediaStream is the component which enables the browser to access media devices, such as microphones, webcams, and speakers. The MediaStream represents a synchronized stream of media data. Each MediaStream has inputs and outputs. The getUserMedia() function is requested to access the user to create and use a local MediaStream.

RTCPeerConnection is a peer connection which handles the peer-to-peer connection between the browsers by sharing the session object. It is used to represent a web real-time communication connection between the users and to handle efficient streaming of data between the two users. The RTCPeerConnection event callback can be used to take care of dealing with the audio/video stream. It can be developed with HyperText Markup Language, version 5 (HTML5) [4].

DataChannel is a channel providing the transport capability to the browser. The DataChannel interface represents a bi-directional data exchange channel between two peers of a connection which includes an incoming and an outgoing stream.

Signaling stage is a process setting up a bidirectional transmission of conversation. Signaling is the exchange of data information between caller and callee connections in the network in order to set up, notify, control, and terminate each connection when a connection is in progress. In addition, the important information that browsers must exchange is the multimedia session description, which includes: the transport information as well as the media type, format, and all associated media configuration parameters necessary to establish the media path.

\section{B. Design of the Telepresence Wheelchair}

We have developed a telepresence wheelchair system based on an electric-powered wheelchair platform modified with many add-on external modules. In order to achieve the desired features, the powered wheelchair is equipped with a Mac mini computer, control components, telecommunication interfaces, touchscreen display, and webcam. In the present paper, in order to perform a two-way audio and video communication, we develop a telepresence wheelchair using a more flexible web real-time communication framework. The architecture of the system is illustrated in Figure 1.

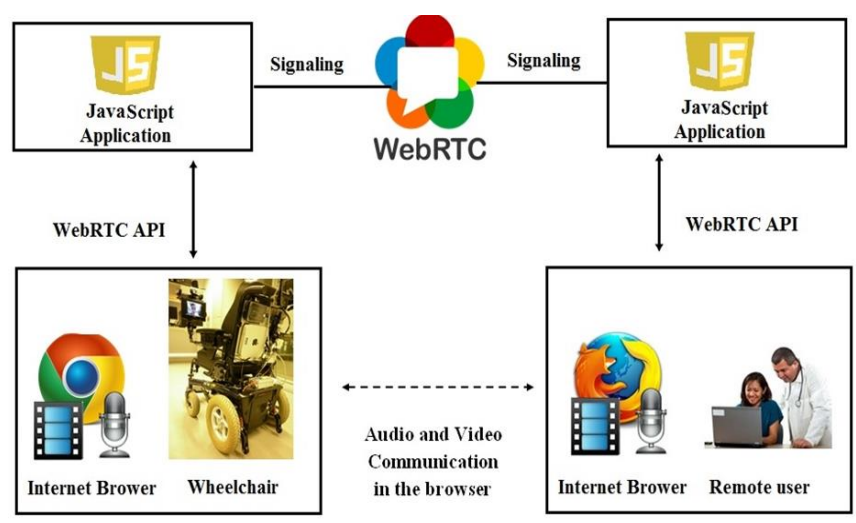

Figure 1. The WebRTC based telepresence wheelchair architecture.

For two-way video communications, a web page with the functions of establishing connections, acquiring, displaying a live video stream and controlling the wheelchair over the Internet from anywhere in real-time has been developed. To be able to achieve the remote interaction characteristics, a control interface with five buttons (move forward, go backward, turn left, turn right, and stop) have been designed to send the control signals from the remote user to the wheelchair over the WebRTC channels. These control signals are written and read by the application programming interfaces developed in JavaScript language and HTML5.

\section{Performance Analysis}

For telepresence systems which are capable of interactive video communication and remotely controllable from a distance, one of the most important design objectives is to transfer voice, video, control signal and data in real-time. The performance of data communications is carefully considered and thoroughly explored to understand the behavior of data transmission. There are many key factors affecting the performance of data transmission including bandwidth, throughput, round-trip-time, and frame rate. In order to evaluate the system streaming performance, these important factors are analyzed.

The bandwidth (W) is closely related to the maximum communication channel capacity in bits per second. In order to determine the maximum rate of transmission data which can be sent over a link, the bandwidth of a communications link is measured. If the bandwidth is sufficient, the packet delay is very low, and interactive media applications work quite well in terms of user satisfaction. The channel capacity is given by the Shannon-Hartley theorem as follows:

$$
C=W \log _{2}\left(1+\frac{S}{N}\right)
$$

where $S / N$ is signal-to-noise ratio. For a system transmitting at maximum capacity, the bandwidth efficiency of the system can be written as

$$
\frac{C}{W}=\log _{2}\left(1+E_{b} \times \frac{C}{N_{o} \times W}\right),
$$


where $E_{b}$ is the average received energy per bit, $N_{0}$ is the noise power density (Watts/Hz). Obviously, the larger the ratio $\frac{C}{W}$, the greater the bandwidth efficiency is.

On the other hand, the throughput is the number of bits transmitted per unit time. A packet contains $L$ bits, and the amount of time devoted to that packet is the actual transmission time $(L / B)$ plus the propagation delay $(d / V)$.

$$
\text { Throughput }=\frac{L}{d / V+L / B} \text {. }
$$

The TCP-Friendly Rate Control (TFRC) bandwidth estimation model is used for bandwidth estimation algorithm, which relies on the $p$-value (the packet loss rate) [9].

$$
T_{T F R C}=\frac{s}{R T T \sqrt{\frac{2 p}{3}}+t_{R T O}\left(\sqrt[3]{\frac{3 p}{8}}\right) p\left(1+32 p^{2}\right)},
$$

where $s$ represents the average size of received packet size per second, $R T T$ is round-trip-time and $t_{R T O}$ is the value of retransmission timer. $T_{T F R C}$ does not represent the actual WebRTC real-time video output rate but it represents an upper bound for it.

To deliver the video with improved quality, we adopted a Google Congestion Control (GCC) algorithm [4]. The sender-side uses the packet loss rate to estimate the sending rate (As) given by

$$
A s(i)=\left\{\begin{array}{lr}
\max \{\mathrm{S}(\mathrm{i}), \mathrm{As}(\mathrm{i}-1)(1-0.5 \mathrm{p}) & \mathrm{p}>0.1 \\
\operatorname{As}(\mathrm{i}-1) & 0.02<\mathrm{p}<0.1 \\
1.05(\mathrm{As}(\mathrm{i}-1)+1 \mathrm{kbps}) & \mathrm{p}<0.02
\end{array}\right.
$$

where $A s(i)$ is the sender available bandwidth estimate at time $\mathrm{i}, S(i)$ is the throughput, and $\mathrm{p}$ is the packet loss rate. The receiver-side estimates the receiving rate given by

$$
\operatorname{Ar}(i)= \begin{cases}\eta \operatorname{Ar}(\mathrm{i}-1) & \text { Increase } \\ \operatorname{Ar}(\mathrm{i}-1) & \text { Hold } \\ \alpha(\mathrm{R}(\mathrm{i}) & \text { Decrease }\end{cases}
$$

where $\operatorname{Ar}(i-1)$ denotes the receiving rate estimate at the time (i-1), $\eta$ is the receiving rate increase constant. $R(i)$ is the current incoming rate, and $\alpha$ represents the incoming rate decrease factor.

\section{EXPERIMENTS AND RESULTS}

The experiments were carried out at various locations where a remote user is a distance away from a wheelchair. The wheelchair was located in the Centre for Health Technologies in the University of Technology Sydney, and the remote user was located in other rooms. The wheelchair battery was fully charged and the speed was reduced to $1 \mathrm{~m} / \mathrm{s}$ to ensure the wireless connection could be maintained. The remote user and wheelchair computer are configured with the Intel Core i7 CPU and 8GB RAM to have sufficient computing power. Window 7 (64bit), Internet browser (Firefox) was installed. The bandwidth management was taken into account to ensure the consistency of the system performance.
We conducted three case studies, and the experiments consisted of 30 trials. We evaluated the performance of the proposed method by implementing WebRTC to stream videos from the wheelchair to a remote location over the Internet with three types of resolution of $320 \times 280,640 \times 480$, and $1024 \times 768$ pixels, respectively. Ten trials were repeated for each resolution. For each trial, the data transmission was observed during the period from 0 to 500 -seconds.

For security reason, the remote user needed to log in the system with username and password. During the experiments, when the connection was established, the remote user could request video communication and control the wheelchair directly. At the remote site, a remote user can hear and observe the environments surrounding the wheelchair. The user interface and the video obtained from the remote user are shown as in Figure 2. Figure 2 illustrates the subjective quality assessments of the video communications by human perception. The results demonstrated that the remote user could obtain a high-quality video and could control the wheelchair with desired directions.

To further examine the system performance, we evaluated the other objective quality measures. The measurement data was recorded and collected carefully. A diagnostic packet and event recording related to the bandwidth estimation, packets sent, round-trip time and frame rates were measured and logged for analysis and evaluation. Table I provides the summary of results of the processing time of the encoded video, the delay and the throughput for each resolution in all trials. It is clearly seen from Table I that the mean (average) encoding time is increased when processing higher resolution videos. Delay during transmission may occur which in turn will result in increasing buffer size for temporary storage, causing longer latency. However, a higher resolution results in more data to be transmitted but does not affect the throughput of the streaming due to congestion control.

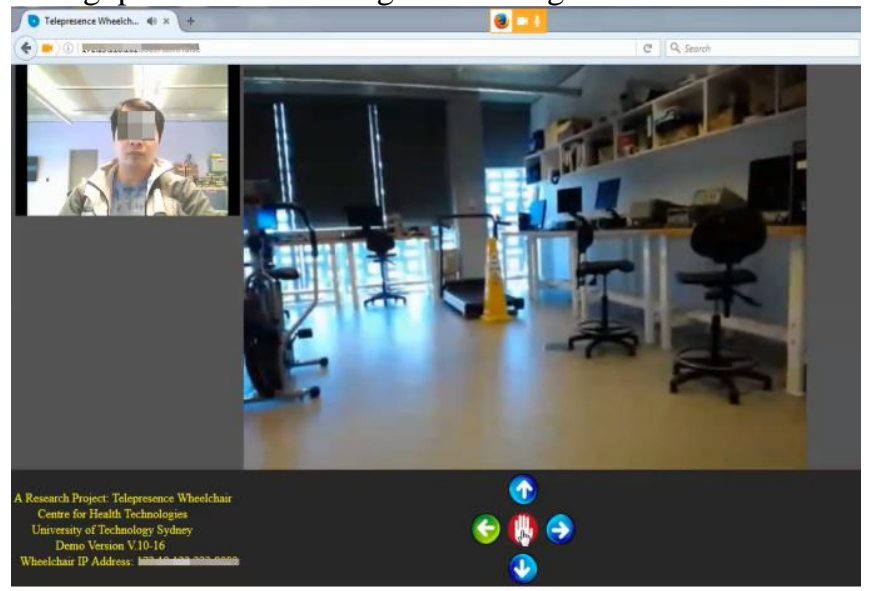

Figure 2. The user interface and the video obtained at the remote user. TABLE I. THE AVERAGE PERFORMANCE RESULTS OF THE 30-TRIALS.

\begin{tabular}{|c|c|c|c|c|}
\hline Platform & $\begin{array}{c}\text { Resolution } \\
\text { (pixels) }\end{array}$ & $\begin{array}{c}\text { Avg. Encode } \\
(\mathbf{m s})\end{array}$ & $\begin{array}{c}\text { Avg. delay } \\
\text { (ms) }\end{array}$ & $\begin{array}{c}\text { Avg. } \\
\text { throughput } \\
\text { (kbps) }\end{array}$ \\
\hline \multirow{3}{*}{ WebRTC } & $320 \times 280$ & $4.00 \pm 0.01$ & $8.06 \pm 0.03$ & 1641.635 \\
\cline { 2 - 5 } & $640 \times 480$ & $7.00 \pm 0.05$ & $12.08 \pm 0.16$ & 1617.374 \\
\cline { 2 - 5 } & $1024 \times 768$ & $11.00 \pm 0.02$ & $18.01 \pm 0.23$ & 1582.916 \\
\hline
\end{tabular}




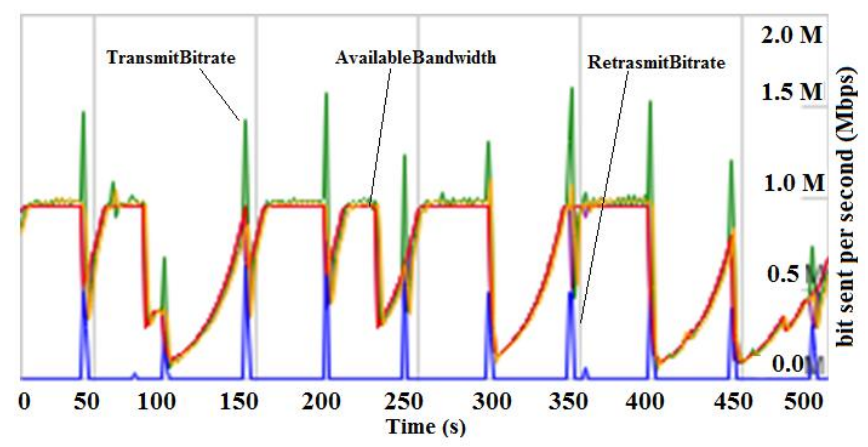

Figure 3. Bandwidth and bitrate of the system.

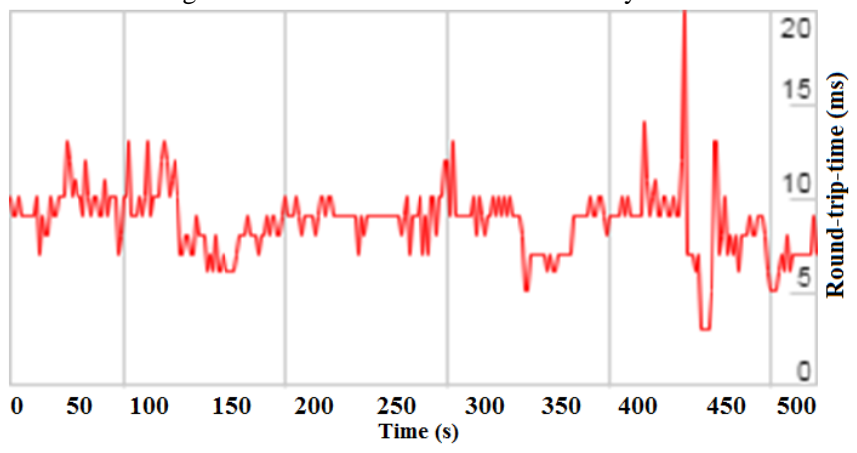

Figure 4. Round-trip time of telepresence based on WebRTC.

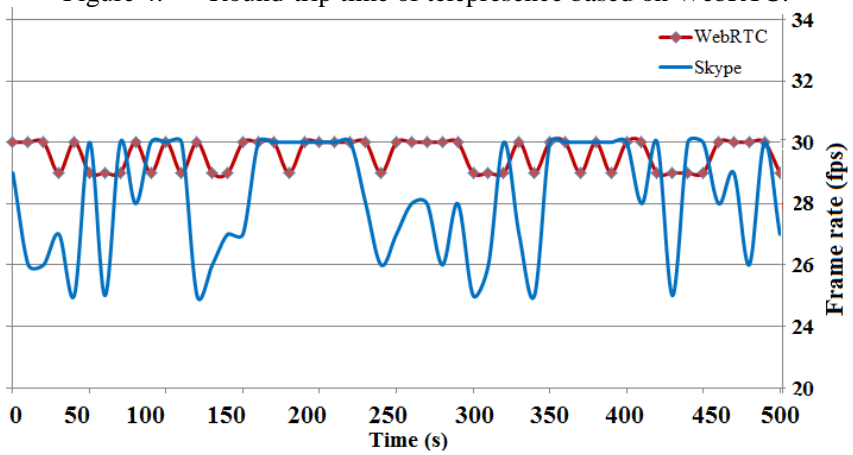

Figure 5. Frame rate sent (fps) of WeRTC vs. Skype.

Figures 3, 4 and 5 are the illustrations of the experimental results of streaming video at a resolution of $640 \times 480$ pixels. The results in Figure 3 show that the packets sending rate adapted with the available bandwidth and reached 1.5 Mbits per second. The results in Figure 4 indicate that the roundtrip-time (RTT) fluctuated from 3 to $20 \mathrm{~ms}$. In addition, it can be observed from Figure 5 that the streaming rate fluctuated and reached a peak rate up to $30 \mathrm{fps}$.

In comparison with [10], it is worth noting that the standard video frame rate is $25 \mathrm{fps}$ (Europe) or $30 \mathrm{fps}$ (North America). Therefore, the video streaming rate of the proposed system has reached the standard of real-time video communication. Regarding the round-trip time, it is worth noting that the RTT of less than $400 \mathrm{~ms}$ is considered as realtime communications [3]. Obviously, the RTTs of WebRTC between two browsers in the experiments is under the RTT threshold value. The RTTs of the experiments are also significantly lower than the results of the recent telepresence robot study, which revealed that $40 \mathrm{~ms}$ delay was considered a good performance [1].

The main findings of the study are the connection time of WebRTC is faster with less latency than Skype platform [13]. The WebRTC processing is done at the user level, which reduces the delay in processing and connection time. Moreover, the transmission rate of WebRTC is able to adapt to the available bandwidth of the network, whereas Skype platform adjusts the video resolution to adapt to the network condition [1-3]. More importantly, WebRTC is open source and easily accessible. The convergence capabilities of the WebRTC technology can provide flexibility for further development and extension. However, the performance may be constrained by the hardware of the system. The video quality relies on the Internet connection bandwidth. It can be improved by using more wireless access points to guarantee the wireless signal. Nevertheless, the development of the telepresence wheelchair using WebRTC has proven to be a potential alternative approach for telepresence system with an open source and low-cost.

\section{CONCLUSION}

In this paper, we have presented a novel telepresence wheelchair using WebRTC. The experiments were carried out to evaluate the system performance. The experimental results are reliable and positive compared to the standard recommendation and other recent approaches based on the traditional platform. The results reveal the promise and potential of WebRTC for such a real-time telepresence wheelchair system. Future work will consider the integration of 360-degree vision and WebRTC for safer navigation of an advanced telepresence wheelchair.

\section{REFERENCES}

[1] M. Denojean-Mairet, Q. Tan, F. Pivot, and M. Ally, "A Ubiquitous Computing Platform - Affordable Telepresence Robot Design and Applications," in 7th International Conference on IEEE Computational Science and Engineering (CSE), 2014, pp. 793-798.

[2] P. Corke, K. Findlater, and E. Murphy, "Skype: a communications framework for robotics" in Proceedings of the 2012 Australasian Conference on Robotics and Automation, 2012.

[3] V. K. L. Ha, T. N. Nguyen, and H. T. Nguyen, "A Telepresence Wheelchair Using Cellular Network Infrastructure in Outdoor Environments," in 38th Annual International Conference of the IEEE Engineering in Medicine and Biology Society (EMBC), 2016, pp. 5352-5355.

[4] R. Atwah, R. Iqbal, S. Shirmohammadi, and A. Javadtalab, "A Dynamic Alpha Congestion Controller for WebRTC," in 2015 IEEE International Symposium on Multimedia (ISM), 2015, pp. 132-135.

[5] C. Y. Chiang, Y. L. Chen, P. S. Tsai, and S. M. Yuan, "A Video Conferencing System Based on WebRTC for Seniors," in 2014 International Conference on Trustworthy Systems and their Applications (TSA), 2014, pp. 51-56.

[6] N. Hongo, H. Yamamoto, and K. Yamazaki, "Web shopping support system for elderly people using WebRTC," in 16th International Conference on Advanced Communication Technology, 2014, pp. 934-940.

[7] V. K. L. Ha, T. N. Nguyen, and H. T. Nguyen, "Real-time transmission of panoramic images for a telepresence wheelchair," in 37th Annual International Conference of the IEEE Engineering in Medicine and Biology Society (EMBC), 2015, pp. 3565-3568.

[8] V. Singh, A. A. Lozano, and J. Ott, "Performance Analysis of Receive-Side Real-Time Congestion Control for WebRTC," in 20th International Packet Video Workshop, 2013, pp. 1-8.

[9] C. Kilinc and K. Andersson, "A Congestion Avoidance Mechanism for WebRTC Interactive Video Sessions in LTE Networks," Wireless Personal Communications, vol. 77, pp. 2417-2443, 2014.

[10] European Telecommunications Standards Institute ETSI, "Review of available material on QoS requirements of Multimedia Services," Telecommunications and Internet converged Services and Protocols for Advanced Networking (TISPAN), 2006. 